\title{
Recurrent Dysphagia Associated with Esophageal Intramural Pseudodiverticulosis
}

\author{
Haider Naqvi ${ }^{a}, b, c, d \quad$ Muhammad Nadeem Yousafa, b, c, d \\ Gavneet Sandhu $^{a, b, c, d}$ Deepty Bhansalie Rehan Farooqi ${ }^{b}$ \\ aDepartment of Medicine, Medstar Union Memorial Hospital, Baltimore, MD, USA; \\ ${ }^{\text {b}}$ Department of Medicine, MedStar Good Samaritan Hospital, Baltimore, MD, USA; \\ 'Department of Medicine, Medstar Franklin Square Medical Center, Baltimore, MD, USA; \\ ${ }^{\mathrm{d}}$ Department of Medicine, MedStar Harbor Hospital, Baltimore, MD, USA; ${ }^{\text {eDepartment of }}$ \\ Pathology, Medstar Franklin Square Medical Center, Baltimore, MD, USA
}

\section{Keywords}

Esophagus · Pseudodiverticulosis · Stricture · Dysphagia

\begin{abstract}
Esophageal intramural pseudodiverticulosis (EIPD) is a rare disorder of the esophagus characterized by the presence of outpouching flask-shaped lesions. These lesions represent false lumens that may be scattered throughout the esophageal wall. We present a rare case of EIPD complicated with esophageal strictures. The case is unique because the small lesions of EIPD remained undiagnosed for many years on prior esophagogastroduodenoscopy examinations until complicated with worsening symptoms of dysphagia due to esophageal stricture. The patient was managed with serial dilations of esophageal stricture. Diagnosing these lesions may be missed in early stages as the lesions are small and may require other radiological modalities to confirm the diagnosis. EIPD lesions itself can be managed conservatively with antiinflammatory therapy and treatment of underlying risk factors. Those with severe disease and coexisting complications are managed with endoscopic or surgical interventions.
\end{abstract}

\section{Introduction}

Esophageal intramural pseudodiverticulosis (EIPD) is a rare benign disease characterized by multiple small flask-shaped outpouchings within the esophageal wall. Pseudodiverticulum is a false outpouching in the mucosal walls as a result of ductal dilatation of submucosal secretory 
glands. Acute on chronic inflammation and concomitant increased intramural pressure around secretory ducts of submucosa is proposed mechanism of diverticula formation. The major difference between a true diverticulum (like Zenker's diverticulum) and pseudodiverticulum is the segments of the mucosal layers that are contained within the out-pouched layers. Pseudodiverticulum contains only mucosal and submucosal layers whereas a true diverticulum contains all layers of the epithelium including the muscle layers. The prevalence rate of EIPD is around $0.15 \%$ amongst patients who underwent esophagram [1]. Whereas, the estimated prevalence rate of true esophageal diverticulosis is 1-3\% in patients with dysphagia [2]. Here, we present a case of a patient who had intermittent dysphagia to solids and liquids for over a decade which was attributed to EIPD after a recent esophagogastroduodenoscopy (EGD).

\section{Case Report/Case Presentation}

A 64-year-old man with gastrointestinal reflux disease (GERD), alcohol use disorder, and recurrent esophageal strictures requiring multiple dilatations in past 4 years presented with recurrent dysphagia after eating solid and semisolid food. He reported a sensation of food getting stuck in his chest and having difficulty with swallowing both solids and semisolids. He denied associated nausea, vomiting, halitosis, odynophagia, night-time awakenings, dyspnea, diaphoresis, fever, chills, or unintentional weight loss. Family history was unremarkable. He has had intermittent dysphagia to solids, semisolids that progressed to liquids over the past 12 years; some resolved spontaneously while others required endoscopic intervention. His last esophageal dilation was in 2-year ago requiring esophageal dilation up to $8 \mathrm{~mm}$ with the use of a pediatric scope. The dysphagia in between endoscopic intervention would occur randomly and over time the symptoms would worsening to the point that he is unable to swallow. When the symptoms would start in between the interventions, he would begin to notice that he is having the sensation of food being stuck but would be able to maintain oral intake, till it would just not tolerable. On presentation, his examination was benign with no pertinent positive findings. On initial laboratory work-up, his electrolytes were within normal range, creatinine $0.80 \mathrm{mg} / \mathrm{dL}$, BUN $13 \mathrm{mg} / \mathrm{dL}$, WBCs $5.6 \mathrm{k} / \mu \mathrm{L}$, and hemoglobin $14.7 \mathrm{~g} / \mathrm{dL}$. No pertinent imaging studies, although previous chest and neck X-rays were normal. Upper endoscopy revealing the presence of food impaction at lower third of esophagus with evidence of reflux esophagitis and multiple EIPD along the middle and lower third of esophagus (shown in Fig. 1). Food impaction was relieved endoscopically, and multiple esophageal biopsies were taken. Patient was discharged on Pantoprazole $40 \mathrm{mg}$ daily for 3 months. Histopathological examination of biopsy demonstrated squamous cell epithelium with acute on chronic inflammation without evidence of malignancy or infections such as fungus or helicobacter on staining (shown Fig. 2). A thorough review of previous endoscopic imaging revealed multiple EPIDs that remained unidentified. Patients continued to experience recurrent dysphagia due to esophageal strictures and required multiple sessions of esophageal dilations on follow-ups.

\section{Discussion}

EIPD is a rare esophageal disease characterized by the presence of numerous small outpouches within the esophageal wall. It was first reported by Mendl in 1960 [1]. Exact etiology of EIPD is unknown; however, studies have shown its association with multiple comorbidities such as diabetes mellitus, esophageal candidiasis, gastroesophageal reflux 
Fig. 1. Esophageal mucosa with multiple scattered indentations revealing the presence of the EIPD found during EGD (white arrow). Measuring $1 \times$ $1 \mathrm{~mm}$. No signs of infection or stricture at this time. EIPD, esophageal intramural pseudodiverticulosis; EGD, esophagogastroduodenoscopy.

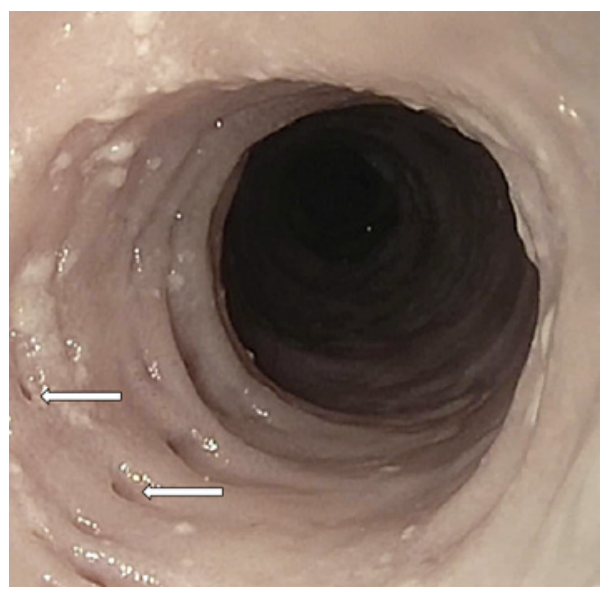

Fig. 2. Histopathological slide of the esophageal biopsy revealing acute on chronic inflammation with reactive epithelial changes. Magnification, $\times 4$.

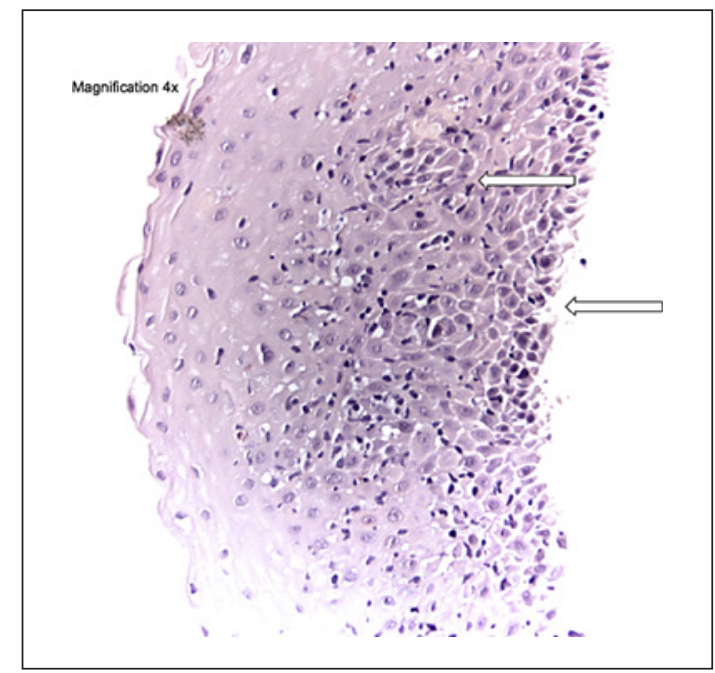

disease, esophageal stenosis, achalasia, Mallory-Weiss syndrome, Crohn's disease, tuberculosis, chronic alcoholism, and corrosive acid injury [3,4]. EIPD is a benign disease in majority of cases; however, esophageal malignancies have also been reported in several case reports $[3,4]$. Disease presentation has a bimodal age distribution known to affect patients in their teen's age or sixth decade of life [5]. No specific gender predilection has been noted, although, in one study they found a gender predilection with male to female ratio of 1.4, respectively [6].

There are no typical symptoms of EIPD. Dysphagia is the most common presenting symptom (80-90\%) in patients with EIPD with an underlying esophageal stricture [5, 7]. In patients with EIPD, dysphagia may be persistent (47\%), intermittent (24\%), or progressive (9\%), while some are asymptomatic $(20 \%)[8,9]$. Patients may have odynophagia as well as symptoms of reflux disease or esophageal stenosis (such as dysphagia to solid and/or liquids and the sense of food particles being stuck in the chest) [5]. In a few patients alarm symptoms such as anorexia, weight loss and even gastrointestinal bleeding can occur [10]. Our patient had underlying risk factors including esophageal stenosis, and GERD that increased his likelihood of developing EIPD. It was unfortunate that EIPD was never reported on prior upper endoscopic evaluations as a result this association was never made. He had been experiencing recurrent episodes of dysphagia for years and was known to have esophageal strictures. For 
his esophageal strictures, he repeatedly had EGD with esophageal dilation for symptomatic relief and proton pump inhibitor therapy without resolution of his symptoms.

Esophageal pseudodiverticulosis has no specific location inclination. The small outpouchings can be found anywhere along the length of the esophagus. Distribution of the outpouching can be diffuse or segmental, and with the segmental distribution, the majority of the outpouches are along the upper third of the esophagus [5,6]. Like benign colonic polyps, patients can have a few $(<5)$ to multiple (up to 20) lesions [4]. These outpouchings range in size from 1 to $4 \mathrm{~mm}$ in diameter and 1-2 $\mathrm{mm}$ in length [1, 5, 6]. Outpouchings are flask-shaped and are spread along the longitudinal axis of the esophagus; at times, the lesions may coalescence leading to the formation of longitudinal tracts [1,11]. A study of 50 patients with EIPD reported longitudinal tracts to be present in $50 \%$ of patients, these tracts can be of varying sizes and appearances; short bridges between 2 pseudodiverticuli to long tracts between multiple pseudodiverticula [11]. Tracts tend to be more prominent in patients with diffuse disease and those that have strictures in the mid-esophagus [11].

Diagnosis of EIPD may be challenging at early stages of disease and a proportion of patients present with complications of disease. The small outpouchings are a few millimeters in size and often go unrecognized on EGD, as evident in our patient. The diagnostic modalities such as those using contrast dye (barium esophagram) have the highest sensitivity to diagnose the lesions $[1,3,4]$. The ingested contrast dye easily involutes into the small lesions and can be detected on imaging. Direct endoscopic visualization demonstrates small openings or linear tracks in the esophageal wall in $20-25 \%$ of patients however diagnosis is rarely made on EGD. Tissue biopsies are typically not required for diagnosis confirmation because biopsies taken during EGD normally show inflammatory changes and the presence of pseudodiverticuli is not noted as outpouches are intramural [5]. In this case, the patient had dysphagia for several years requiring recurrent dilatation for his esophageal stricture. Until the latest EGD when the EIPD was visualized the lesions were missed on the previous EGD. On reevaluation of the previous EGD images, these lesions were noted. This case illustrates the diagnostic challenge posed by EIPD. If the patient had undergone contrast studies in addition to EGD's, the cause of his dysphagia may have been discovered earlier.

Mainstay of treatment is the symptomatic management, control of the associated comorbidities, and prevention of complications such as esophageal stricture, perforation, and infections [7]. Of those diagnosed with EIPD, approximately $10 \%$ of patients do not require any treatments [5]. Those with underlying GERD would have relief of their symptoms with PPI therapy. However, in patients with evident esophageal strictures, such as our patient, serial endoscopic dilation of esophageal strictures lead to improvement in symptoms $[5,7,12,13]$. In one study, it was reported that management of the esophageal strictures can lead to reduction or complete resolution of the pseudodiverticuli likely due to relieve of intramural esophageal pressure proximal to the stricture and a resolution of esophageal submucosal secretory gland inflammation [14]. Whereas, long-term observational studies have shown no regression of the pseudodiverticuli overtime on follow up EGD despite optimal medical management of symptoms [7]. Our patient required serial EGD dilatations for symptomatic relief on several follow-ups. In cases with extensive disease or those with complicated EIPD refractory to medical management, surgical intervention such as esophagectomy can be pursued [15].

The full implications of EIPD are still unknown, beyond the symptoms of dysphagia several studies reported association of EIPD with esophageal malignancy in $4.5 \%$ of cases [3, 5]. Other complications of untreated EIPD are bleeding, perforation, fistula formation, and mediastinal abscesses [7]. Currently, there are no guidelines recommendations for gastroenterologists regarding the frequency of performing interval EGDs in a patient with EIPD [3]. Currently, there are no set guidelines for the surveillance of EIPD for esophageal carcinoma;

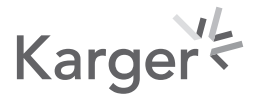


therefore, further prospective studies are required to determine the value of interval endoscopic surveillance of these patients for the development of carcinoma.

EIPD is a rare esophageal disease of unclear etiology presenting as chronic progressive dysphagia. The exact sensitivity and specificity of diagnostic tools are unknown; however, barium esophagram tends to yield higher sensitivities. EIPD is managed with symptomatic treatment of underlying conditions as there is no evidence of complete regression of pseudodiverticuli in the medical literature. Internists and gastroenterologists need to consider EIPD as part of their differential for intermittent, progressive, and chronic dysphagia as patients may also have underlying esophageal strictures that may progress to esophageal carcinomas in certain cases. Currently, there are no guidelines to guide gastroenterologists regarding the frequency intervals of performing EGDs in a patient with EIPD. Although there are no set guidelines for surveying patients with EIPD for esophageal carcinoma, further prospective studies are required to determine the value of interval endoscopic surveillance of these patients for the development of carcinoma. Overall, this case illustrates how esophageal intramural pseudodiverticuli pose a diagnostic and therapeutic challenge.

\section{Acknowledgement}

This manuscript does not include any nonauthor contributors to acknowledge.

\section{Statement of Ethics}

Based up IRB guidelines at our institution, case reports do not require IRB review and/ or approval. Informed written consent was obtained from the patient for use of the patient's information and the images for use in the manuscript for publication. It was conveyed to the patient that all patient identifiers have been removed.

\section{Conflict of Interest Statement}

The authors of this manuscript do not have any conflicts of interest to declare.

\section{Funding Sources}

This manuscript did not receive any funding.

\section{Author Contributions}

Haider Naqvi, Muhammad Yousaf, and Gavneet Sandhu were handled the manuscript writing, proofreading, figures, and review of data. Deepty Bhansali helped obtained the histology slides and labeling of the figures along with the legend of the figures. Rehan Farooqi provided overall supervision and proofreading.

\section{Karger'k}


Naqvi et al.: Recurrent Dysphagia with EIPD

\section{References}

1 Levine MS, Moolten DN, Herlinger H, Laufer I. Esophageal intramural pseudodiverticulosis: a reevaluation. AJR Am J Roentgenol. 1986;147:1165-70.

2 Little RE, Bock JM. Pharyngoesophageal diverticuli: diagnosis, incidence and management. Curr Opin Otolaryngol Head Neck Surg. 2016;24(6):500-4.

3 Ali 0, Asumu H, Kaur T, Mathew A, Kim R. A rare cause of dysphagia due to esophageal intramural pseudodiverticulosis: a case report and review of literature. BMC Gastroenterol. 2020;20(1):72.

4 Szczesna M, Gatarek J, Orlowski T. Esophageal intramural pseudodiverticulosis as a diagnostic and therapeutic problem. Kardiochir Torakochirurgia Pol. 2016;13(3):265-8.

5 Chon YE, Hwang S, Jung KS, Lee HJ, Lee SG, Shin SK, et al. A case of esophageal intramural pseudodiverticulosis. Gut Liver. 2011;5(1):93-5.

6 Stephens WP, Mossman A, Ratcliffe JF, Gould DA, Oleesky S. Intramural pseudodiverticulosis: an unusual cause of benign oesophageal stricture. Postgrad Med J. 1986;62:201-4.

7 de Oliveira LL, Carneiro FO, Baba ER, Vilaça TG, Chaves DM, Artifon EL, et al. Esophageal intramural pseudodiverticulosis: a rare endoscopic finding. Case Rep Med. 2013;2013:154767.

8 Flora KD, Gordon MD, Lieberman D, Schmidt W. Esophageal intramural pseudodiverticulosis. Dig Dis. 1997; 15(1-2):113-9.

9 Teraishi F, Fujiwara T, Jikuhara A, Kamitani S, Morino Y, Sato K, et al. Esophageal intramural pseudodiverticulosis with esophageal strictures successfully treated with dilation therapy. Ann Thorac Surg. 2006;82(3): 1119-21.

10 Hahne M, Schilling D, Arnold JC, Riemann JF. Esophageal intramural pseudodiverticulosis: review of symptoms including upper gastrointestinal bleeding. J Clin Gastroenterol. 2001;33(5):378-82.

11 Canon CL, Levine MS, Cherukuri R, Johnson LF, Smith JK, Koehler RE. Intramural tracking: a feature of esophageal intramural pseudodiverticulosis. AJR Am J Roentgenol. 2000;175:371.

12 Cho SR, Sanders MM, Turner MA, Liu CI, Kipreos BE. Esophageal intramural pseudodiverticulosis. Gastrointest Radiol. 1981;6(1):9-16.

13 Van Laer W, Urbain D, Reynaert H. Esophageal intramural pseudodiverticulosis. Clin Gastroenterol Hepatol. 2007;5(12):A22.

14 Kochhar R, Mehta SK, Nagi B, Goenka MK. Corrosive acid-induced esophageal intramural pseudodiverticulosis. A study of 14 patients. J Clin Gastroenterol. 1991;13(4):371-5.

15 Liu SM, Wu HH, Chang KK, Tseng LJ, Han SC, Mo LR. Esophageal intramural pseudodiverticulosis complicated with stricture. J Formos Med Assoc. 2010;109(3):241-4. 